\title{
Liouville theorems for $F$-harmonic maps and their applications
}

\author{
Yuxin Dong*, Hezi Lin and Guilin Yang
}

\begin{abstract}
We prove several Liouville theorems for $F$-harmonic maps from some complete Riemannian manifolds by assuming some conditions on the Hessian of the distance function, the degrees of $F(t)$ and the asymptotic behavior of the map at infinity. In particular, the results can be applied to $F$-harmonic maps from some pinched manifolds, and can deduce a Bernstein type result for an entire minimal graph.
\end{abstract}

\section{Introduction}

In $[\mathrm{Ar}], \mathrm{M}$. Ara introduced the $F$-harmonic map and its associated stress-energy tensor. The concept of $F$-harmonic maps unifies the concepts of harmonic maps, $p$ harmonic maps, minimal hypersurfaces, maximal spacelike hypersurfaces and steady compressible flows, etc. It is known that the stress-energy tensor is a useful tool for studying the energy behavior and vanishing results of related functional (cf. [DW]).

Liouville type theorems for harmonic maps, $p$-harmonic maps and $F$-harmonic maps were investigated by several authors (cf. [GRSB], [Ch], [Hi], [SY], [Se], [Ji], [DW] and the references therein). Up to now, most Liouville results have been established by assuming either the finiteness of the energy of the map or the smallness of the whole image of the domain manifold under the map. In [Ji], Z.R. Jin proved several interesting Liouville theorems for harmonic maps from complete manifolds, whose assumptions concern the asymptotic behavior of the maps at infinity. One special case of his results is that if $u:\left(R^{m}, g_{0}\right) \rightarrow\left(N^{n}, h\right)$ is a harmonic map, and $u(x) \rightarrow p_{0} \in N^{n}$ as $|x| \rightarrow \infty$, then $u$ is a constant map.

In this paper, we generalize Jin's method and results to $F$-harmonic maps. The procedure consists of two steps. The first step is to use the $F$-stress energy tensor, by choosing a suitable vector field, to deduce the lower energy rates of the $F$-harmonic

2010 Mathematics Subject Classification. Primary: 35B53, 58Z05, 53C43.

*Supported by NSFC grant No 10971029, and NSFC-NSF grant No 1081112053.

Key words and phrases. complete Riemannian manifold, F-harmonic map, Liouville theorem. 
maps. The second step is to use the asymptotic assumption of the maps at infinity to obtain the upper energy growth rates of the $F$-harmonic maps. Under suitable conditions on $F$ and the Hessian of the distance functions of the domain manifolds, one may show that these two growth rates are contradictory unless the $F$-harmonic maps are constant maps. In this way, we establish some Liouville results for $F$-harmonic maps with the asymptotic property at infinity from some complete manifolds (cf. $\S 5$ for detailed statements). Finally, in $\S 6$, we show that the asymptotic conditions on $F$-harmonic maps for Liouville theorems can be relaxed if the target manifold is more special. These Liouville theorems enable us to give an interesting application for a global minimal graphic hypersurface $(x, u(x))$ in $R^{m+1}$ as follows: If there exists a constant $c$ and $\lim _{R \rightarrow \infty|x|=R}\left\{\frac{(u-c)^{2}}{\sqrt{1+|d u|^{2}}}\right\}=0$, then the graph is a horizontal hyperplane.

This paper is organized as follows. In $\S 2$, we present some basic notions, some examples of $F$-harmonic maps and a useful integral formula associated with the $F$ stress energy tensor. In $\S 3$ and $\S 4$, under suitable conditions on the domain manifolds and the asymptotic condition on the maps, we derive the lower energy growth rates and the upper energy growth rates for $F$-harmonic maps respectively. In $\S 5$ and $\S 6$, we establish the main Liouville results and give some applications.

\section{Preliminaries}

Let $F:[0, \alpha) \rightarrow[0, \infty)$ be a $C^{2}$ function with $F(0)=0$ such that $F^{\prime}>0$ on $(0, \alpha)$ for some $\alpha>0$. For a smooth map $u:(M, g) \rightarrow(N, h)$ between Riemannian manifolds $(M, g)$ and $(N, h)$, we define the $F$-energy $E_{F}(u)$ by

$$
E_{F}(u)=\int_{M} F\left(\frac{|d u|^{2}}{2}\right) d v_{g}=\int_{M} F\left(\frac{1}{2} h_{\alpha \beta} \frac{\partial u^{\alpha}}{\partial x_{i}} \frac{\partial u^{\beta}}{\partial x_{j}} g^{i j}\right) d v_{g}
$$

The map $\mathrm{u}$ is called $F$-harmonic if it is a critical point of the functional $E_{F}(u)$, that is,

$$
\frac{d}{d t} E_{F}\left(u_{t}\right)=0
$$

for any compactly supported variation $u_{t}: M \rightarrow N(-\epsilon<t<\epsilon)$ with $u_{0}=u$. the $F$-tension field $\tau_{F}(u)$ of $u$ is defined by

$$
\tau_{F}(u)=F^{\prime}\left(\frac{|d u|^{2}}{2}\right) \tau(u)+u_{*}\left(\operatorname{grad}\left(F^{\prime}\left(\frac{|d u|^{2}}{2}\right)\right)\right) .
$$

From $[\mathrm{Ar}]$, we know that $u$ is $F$-harmonic if and only if $\tau_{F}(u)=0$.

Example 2.1. (cf. [Ar], [DW]) When $F(t)=2 t, \frac{1}{p}(2 t)^{p / 2},(1+2 t)^{\alpha}(\alpha>1$, $\operatorname{dim} M=2)$, and $e^{2 t}$, the $F$-harmonic map becomes a harmonic map, a $p$-harmonic map, an $\alpha$-harmonic map, and an exponentially harmonic map respectively. 
Example 2.2. (cf. [Ya], [DW]) Let $\left.M^{m}=(x, u(x))\right) \hookrightarrow R^{m+1}$ be a graph defined on $R^{m}$, where $u: R^{m} \rightarrow R$ be a smooth function. Then $M$ is a minimal graph if and only if $u: R^{m} \rightarrow R$ is a $F$-harmonic map with $F(t)=\sqrt{1+2 t}-1$.

Example 2.3. (cf. $[\mathrm{Ot} 1,2]$ ) Set $F(t)=\int_{0}^{2 t} \rho(s) d s$ with $\rho: R^{+} \cup\{0\} \rightarrow R^{+}$. Under suitable conditions on $\rho$, the $F$-harmonic maps have a physical analogy as steady compressible flows on a Riemannian manifold.

From now on, we will assume that $F$ is defined on $[0, \infty)$, that is, $\alpha=\infty$. Similar to $[\mathrm{Ka}]$ and $[\mathrm{DW}]$, we may define the upper degree $d_{F}$ and the lower degree $l_{F}$ of $F$ as follows:

$$
d_{F}=\sup _{t \geq 0} \frac{t F^{\prime}(t)}{F(t)}
$$

and

$$
l_{F}=\inf _{t \geq 0} \frac{t F^{\prime}(t)}{F(t)} .
$$

For example, if $F(t)=\frac{1}{p}(2 t)^{p / 2}$, then $d_{F}=l_{F}=p / 2$; if $F(t)=\sqrt{1+2 t}-1$, then $d_{F}=1$ and $d_{l}=\frac{1}{2}$. In general, we have $l_{F} \leq d_{F}$. From now on, we always assume that $d_{F}<+\infty$ and $m>\max \left\{2,2 d_{F}\right\}$. The stress-energy tensor associated with the functional $E_{F}(u)$ is defined by $([\mathrm{Ar}])$

$$
S_{F}(u)=F\left(\frac{|d u|^{2}}{2}\right) g-F^{\prime}\left(\frac{|d u|^{2}}{2}\right) u^{*}(h) .
$$

From [Ar], we know that if $u$ is $F$-harmonic, then $\operatorname{div} S_{F}(u)=0$. Recall that for a two tensor field $T \in \Gamma\left(T^{*} M \otimes T^{*} M\right)$, it is divergence $\operatorname{div} T \in \Gamma\left(T^{*} M\right)$ is defined by

$$
(\operatorname{div} T)(X)=\sum_{i}\left(\nabla_{e_{i}} T\right)\left(e_{i}, X\right)
$$

where $\left\{e_{i}\right\}$ is an orthonormal basis of $T M$. For a vector field $X$ on $M$, its dual one form $\theta_{X}$ is given by

$$
\theta_{X}(Y)=g(X, Y)
$$

The covariant derivative of $\theta_{X}$ gives a 2-tensor field $\nabla \theta_{X}$ :

$$
\left(\nabla \theta_{X}\right)(Y, Z)=\left(\nabla_{Z} \theta_{X}\right)(Y)=g\left(\nabla_{Z} X, Y\right), \quad \forall Y, Z \in T M .
$$

If $X=\nabla \psi$ is the gradient of some smooth function $\psi$ on $M$, then $\theta_{X}=d \psi$ and $\nabla \theta_{X}=\operatorname{Hess}(\psi)$.

Lemma 2.1. ([Ba], $[D W])$ Let $T$ be a symmetric (0,2)-type tensor field and let $X$ be a vector field, then

$$
\begin{aligned}
\operatorname{div}\left(i_{X} T\right) & =(\operatorname{div} T)(X)+\left\langle T, \nabla \theta_{X}\right\rangle \\
& =(\operatorname{div} T)(X)+\frac{1}{2}\left\langle T, L_{X} g\right\rangle
\end{aligned}
$$


Proof. Let $\left\{e_{i}\right\}$ be a local orthonormal frame field around a point $p$ such that $\left(\nabla e_{i}\right)_{p}=$ 0 . Then

$$
\begin{aligned}
\operatorname{div}\left(i_{X} T\right) & =\sum_{i=1}^{m}\left(\nabla_{e_{i}}\left(i_{X} T\right)\right)\left(e_{i}\right) \\
& =\sum_{i=1}^{m}\left(\nabla_{e_{i}}\left(T\left(X, e_{i}\right)\right)-T\left(X, \nabla_{e_{i}} e_{i}\right)\right) \\
& =(\operatorname{div} T)(X)+\sum_{i, j=1}^{m} T\left(e_{i}, e_{j}\right)\left\langle\nabla_{e_{i}} X, e_{j}\right\rangle \\
& =(\operatorname{div} T)(X)+\left\langle T, \nabla \theta_{X}\right\rangle .
\end{aligned}
$$

We also have

$$
\begin{aligned}
\left\langle L_{X} g, T\right\rangle & =\sum_{i, j}\left(L_{X} g\right)\left(e_{i}, e_{j}\right) T\left(e_{i}, e_{j}\right) \\
& =\sum_{i, j}\left\{X g\left(e_{i}, e_{j}\right)-g\left(\left[X, e_{i}\right], e_{j}\right)-g\left(e_{i},\left[X, e_{j}\right]\right)\right\} T\left(e_{i}, e_{j}\right) \\
& =\sum_{i, j} 2 g\left(\nabla_{e_{i}} X, e_{j}\right) T\left(e_{i}, e_{j}\right) \\
& =2\left\langle T, \nabla \theta_{X}\right\rangle .
\end{aligned}
$$

Therefore (2.6) and (2.7) yield this lemma.

Let $D$ be any bounded domain of $M$ with $C^{1}$ boundary. By applying Lemma 2.1 to $S_{F}$ and using the divergence theorem, we immediately have the following integral formula (see $[\mathrm{Xi}],[\mathrm{DW}])$ :

$$
\int_{\partial D} S_{F}(u)(X, \nu) d s_{g}=\int_{D}\left[\left\langle S_{F}(u), \frac{1}{2} L_{X} g\right\rangle+\left(\operatorname{div} S_{F}(u)\right)(X)\right] d v_{g}
$$

where $\nu$ is the unit outward normal vector field along $\partial D$. In particular, if $u$ is a $F$-harmonic map, then by $\operatorname{div} S_{F}(u)=0$, we have

$$
\int_{\partial D} S_{F}(u)(X, \nu) d s_{g}=\int_{D}\left\langle S_{F}(u), \frac{1}{2} L_{X} g\right\rangle d v_{g} .
$$

\section{Lower energy growth rates for $F$-harmonic maps}

Let $\left(M^{m}, g_{0}\right)$ be a complete Riemannian manifold with a pole $x_{0}$. Denote by $r(x)$ the $g_{0}$-distance function relative to the pole $x_{0}$, that is, $r(x)=\operatorname{dist}_{g_{0}}\left(x, x_{0}\right)$. Set $B(r)=\left\{x \in M^{m}: r(x) \leq r\right\}$. It is known that $\frac{\partial}{\partial r}$ is always an eigenvector of Hess $_{g_{0}}\left(r^{2}\right)$ associated to eigenvalue 2. Denote by $\lambda_{\max }$ (resp. $\lambda_{\min }$ ) the maximum (resp. minimal ) eigenvalues of $\operatorname{Hess}_{g_{0}}\left(r^{2}\right)-2 d r \otimes d r$ at each point of $M \backslash\left\{x_{0}\right\}$.

From now on, we consider an $F$-harmonic map $u:\left(M^{m}, g\right) \rightarrow(N, h)$, where $g=f^{2} g_{0}, 0<f \in C^{\infty}(M)$. Clearly the vector field

$$
\nu=f^{-1} \frac{\partial}{\partial r}
$$


is an outer unit normal vector field along $\partial B(r) \subset(M, g)$.

Henceforth we will assume that $f$ satisfies either $\frac{\partial \log f}{\partial r} \geq 0$ or $\frac{\partial \log f}{\partial r} \leq 0$ on $M \backslash\left\{x_{0}\right\}$. The further conditions for $f$ are as follows:

$\left(f_{1}\right)$ if $\frac{\partial \log f}{\partial r} \geq 0$ (resp. $\left.\frac{\partial \log f}{\partial r} \leq 0\right)$, there is a constant $\sigma>0$ such that

$$
\begin{gathered}
\left(m-2 d_{F}\right) r \frac{\partial \log f}{\partial r}+\frac{(m-1)}{2} \lambda_{\min }+1-d_{F} \max \left\{2, \lambda_{\max }\right\} \geq \sigma \\
\left(\text { resp. }\left(m-2 l_{F}\right) r \frac{\partial \log f}{\partial r}+\frac{(m-1)}{2} \lambda_{\min }+1-d_{F} \max \left\{2, \lambda_{\max }\right\} \geq \sigma\right) .
\end{gathered}
$$

$\left(f_{2}\right)$ there are constants $C>0, R_{0}>0$ such that

$$
\left(\int_{R}^{\infty} \frac{d r}{\int_{\partial B(r)} f^{m-2}(x) d s_{g_{0}}}\right)^{-1} \leq C R^{\sigma} \quad \text { for } \quad R>R_{0}
$$

where $\sigma$ is the constant in $\left(f_{1}\right)$.

$\left(f_{3}\right)$ there are constants $C>0, R_{0}>0$, such that

$$
\int_{\partial B(R)} f^{m-2}(x) d s_{g_{0}} \leq C R \log R \text { for } R>R_{0} .
$$

Now we take $X=r \frac{\partial}{\partial r}=\frac{1}{2} \nabla^{0} r^{2}$ in (2.9), where $\nabla^{0}$ denotes the covariant derivative determined by the metric $g_{0}$. By a direct computation, we have

$$
\begin{aligned}
\frac{1}{2} L_{X} g & =\frac{1}{2} L_{r \frac{\partial}{\partial r}} f^{2} g_{0} \\
& =r f \frac{\partial f}{\partial r} g_{0}+f^{2}\left\{\frac{1}{2} L_{X} g_{0}\right\} \\
& =r \frac{\partial \log f}{\partial r} g+\frac{1}{2} f^{2} L_{X} g_{0}
\end{aligned}
$$

and thus

$$
\begin{aligned}
g\left(S_{F}(u), \frac{1}{2} L_{X} g\right) & =g\left(S_{F}(u), r \frac{\partial \log f}{\partial r} g+\frac{1}{2} f^{2} L_{X} g_{0}\right) \\
& =r \frac{\partial \log f}{\partial r} g\left(S_{F}(u), g\right)+\frac{1}{2} f^{2} g\left(S_{F}(u), \operatorname{Hess}_{g_{0}}\left(r^{2}\right)\right) .
\end{aligned}
$$

Using (2.4), we have

$$
\left(m-2 l_{F}\right) F\left(\frac{|d u|^{2}}{2}\right) \geq g\left(S_{F}(u), g\right) \geq\left(m-2 d_{F}\right) F\left(\frac{|d u|^{2}}{2}\right) .
$$

Let $\left\{e_{i}\right\}_{i=1}^{m}$ be an orthonormal basis with respect to $g_{0}$ and $e_{m}=\frac{\partial}{\partial r}$. We may assume that $\operatorname{Hess}_{g_{0}}\left(r^{2}\right)$ becomes a diagonal matrix w.r.t. $\left\{e_{i}\right\}$. Then $\left\{\tilde{e}_{i}=f^{-1} e_{i}\right\}_{i=1}^{m}$ is an orthonormal basis with respect to $g$. 


$$
\begin{aligned}
f^{2} g\left(S_{F}(u), \text { Hess }_{g_{0}}\left(r^{2}\right)\right)= & f^{2} \sum_{i, j=1}^{m} S_{F}(u)\left(\tilde{e}_{i}, \tilde{e}_{j}\right) \operatorname{Hess}_{g_{0}}\left(r^{2}\right)\left(\tilde{e}_{i}, \tilde{e}_{j}\right) \\
= & f^{2}\left\{\sum_{i=1}^{m} F\left(\frac{|d u|^{2}}{2}\right) \operatorname{Hess}_{g_{0}}\left(r^{2}\right)\left(\tilde{e}_{i}, \tilde{e}_{i}\right)\right. \\
& \left.-\sum_{i, j=1}^{m} F^{\prime}\left(\frac{|d u|^{2}}{2}\right)\left\langle d u\left(\tilde{e}_{i}\right), d u\left(\tilde{e}_{j}\right)\right\rangle \operatorname{Hess}_{g_{0}}\left(r^{2}\right)\left(\tilde{e}_{i}, \tilde{e}_{j}\right)\right\} \\
= & F\left(\frac{|d u|^{2}}{2}\right) \triangle_{g_{0}} r^{2} \\
& -F^{\prime}\left(\frac{|d u|^{2}}{2}\right) \sum_{i=1}^{m}\left\langle d u\left(\tilde{e}_{i}\right), d u\left(\tilde{e}_{i}\right)\right\rangle \operatorname{Hess}_{g_{0}}\left(r^{2}\right)\left(e_{i}, e_{i}\right)
\end{aligned}
$$

Using the definition of the upper degree, we have

$$
\begin{aligned}
f^{2} g\left(S_{F}(u), \text { Hess }_{g_{0}}\left(r^{2}\right)\right) \geq & {\left[(m-1) \lambda_{\min }+2\right] F\left(\frac{|d u|^{2}}{2}\right) } \\
& -\max \left\{2, \lambda_{\max }\right\} F^{\prime}\left(\frac{|d u|^{2}}{2}\right) \sum_{i=1}^{m}\left\langle d u\left(\tilde{e}_{i}\right), d u\left(\tilde{e}_{i}\right)\right\rangle \\
\geq & {\left[(m-1) \lambda_{\min }+2-2 d_{F} \max \left\{2, \lambda_{\max }\right\}\right] F\left(\frac{|d u|^{2}}{2}\right) . }
\end{aligned}
$$

In following we only consider the case $\frac{\partial \log f}{\partial r} \geq 0$, because the argument for the other case $\frac{\partial \log f}{\partial r} \leq 0$ is similar.

Combining (3.2), (3.3) and (3.5), we have

$$
\begin{aligned}
g\left(S_{F}(u), \frac{1}{2} L_{X} g\right) \geq & {\left[\left(m-2 d_{F}\right) r \frac{\partial \log f}{\partial r}+\frac{(m-1)}{2} \lambda_{\min }+1\right.} \\
& \left.-d_{F} \max \left\{2, \lambda_{\max }\right\}\right] F\left(\frac{|d u|^{2}}{2}\right) .
\end{aligned}
$$

By the coarea formula and $|\nabla r|=f^{-1}$, we deduce that

$$
\begin{aligned}
\int_{\partial B(r)} S_{F}(u)(X, \nu) d s_{g} & \leq \int_{\partial B(r)} F\left(\frac{|d u|^{2}}{2}\right) g\left(r \frac{\partial}{\partial r}, \nu\right) d s_{g} \\
& =r \int_{\partial B(r)} F\left(\frac{|d u|^{2}}{2}\right) f d s_{g} \\
& =r \frac{d}{d r} \int_{0}^{r}\left\{\frac{\int_{\partial B(t)} F\left(\frac{|d u|^{2}}{2}\right) d s_{g}}{|\nabla r|}\right\} d t \\
& =r \frac{d}{d r} \int_{B(r)} F\left(\frac{|d u|^{2}}{2}\right) d v_{g} .
\end{aligned}
$$

Hence, by (2.9), (3.6) and (3.7), we have

$$
\begin{aligned}
r \frac{d}{d r} \int_{B(r)} F\left(\frac{|d u|^{2}}{2}\right) d v_{g} \geq & \int_{B(r)}\left[\left(m-2 d_{F}\right) r \frac{\partial \log f}{\partial r}+\frac{(m-1)}{2} \lambda_{\min }+1\right. \\
& \left.-d_{F} \max \left\{2, \lambda_{\max }\right\}\right] F\left(\frac{|d u|^{2}}{2}\right) d v_{g}
\end{aligned}
$$


By $\left(f_{1}\right)$, there is a constants $\sigma>0$ such that

$$
\left(m-2 d_{F}\right) r \frac{\partial \log f}{\partial r}+\frac{(m-1)}{2} \lambda_{\min }+1-d_{F} \max \left\{2, \lambda_{\max }\right\} \geq \sigma,
$$

thus

$$
r \frac{d}{d r} \int_{B(r)} F\left(\frac{|d u|^{2}}{2}\right) d v_{g} \geq \sigma \int_{B(r)} F\left(\frac{|d u|^{2}}{2}\right) d v_{g}
$$

i.e.,

$$
\frac{d}{d r} \frac{\int_{B(r)} F\left(\frac{|d u|^{2}}{2}\right) d v_{g}}{r^{\sigma}} \geq 0
$$

Therefore

$$
\frac{\int_{B\left(\rho_{1}\right)} F\left(\frac{|d u|^{2}}{2}\right) d v_{g}}{\rho_{1}^{\sigma}} \leq \frac{\int_{B\left(\rho_{2}\right)} F\left(\frac{|d u|^{2}}{2}\right) d v_{g}}{\rho_{2}^{\sigma}}
$$

for $0<\rho_{1} \leq \rho_{2}$.

From the above discussion, we can get the following theorem.

Proposition 3.1. Let $u:\left(M^{m}, f^{2} g_{0}\right) \rightarrow(N, h)$ be a $C^{2} F$-harmonic map. Suppose $f$ satisfies $\left(f_{1}\right)$. If $u$ is not a constant map, then we have the following estimate

$$
\int_{B(R)} F\left(\frac{|d u|^{2}}{2}\right) d v_{g} \geq c(u) R^{\sigma} \quad \text { as } R \rightarrow \infty
$$

where $c(u)>0$ is a constant only depending on $u$.

Next, we show that if the $F$-harmonic has the unique continuation property(e.g., $F(t)=t, \sqrt{1+2 t}-1$ in examples 2.2 , etc.), the condition $\left(f_{1}\right)$ in Proposition 3.1 may be replaced by:

$\left(\tilde{f}_{1}\right)$ The left hand sides of the inequalities in $\left(f_{1}\right)$ are nonnegative on the whole $M^{m}$ and there exists an $R_{0}>0$ such that $\left(f_{1}\right)$ holds for $r(x) \geq R_{0}$.

Assuming $\left(\tilde{f}_{1}\right)$, taking $X=r \frac{\partial}{\partial r}$ and applying Lemma 2.1 to $\operatorname{div}\left(i_{X} S_{F}(u)\right)$ on $B(R) \backslash B\left(R_{0}\right)$, we get

$$
\begin{aligned}
& \int_{\partial(R)} S_{F}(u)(X, \nu) d s_{g}-\int_{\partial\left(R_{0}\right)} S_{F}(u)(X, \nu) d s_{g} \\
& =\int_{B(R) \backslash B\left(R_{0}\right)} g\left(S_{F}(u), \frac{1}{2} L_{X} g\right) d v_{g} \\
& \geq \int_{B(R) \backslash B\left(R_{0}\right)}\left[\left(m-2 d_{F}\right) r \frac{\partial \log f}{\partial r}+\frac{(m-1)}{2} \lambda_{\min }+1\right. \\
& \left.\quad-d_{F} \max \left\{2, \lambda_{\max }\right\}\right] F\left(\frac{|d u|^{2}}{2}\right) d v_{g} .
\end{aligned}
$$

Set $H\left(R_{0}\right)=\int_{\partial\left(R_{0}\right)} S_{F}(u)(X, \nu) d s_{g}$, then by (3.7) and (3.13), we have

$$
R \frac{d}{d R} \int_{B(R)} F\left(\frac{|d u|^{2}}{2}\right) d s_{g}-H\left(R_{0}\right) \geq \sigma \int_{B(R) \backslash B\left(R_{0}\right)} F\left(\frac{|d u|^{2}}{2}\right) d v_{g},
$$


and thus

$$
R \frac{d}{d R}\left\{\int_{B(R) \backslash B\left(R_{0}\right)} F\left(\frac{|d u|^{2}}{2}\right) d v_{g}+\frac{H\left(R_{0}\right)}{\sigma}\right\} \geq \sigma\left\{\int_{B(R) \backslash B\left(R_{0}\right)} F\left(\frac{|d u|^{2}}{2}\right) d v_{g}+\frac{H\left(R_{0}\right)}{\sigma}\right\} .
$$

To get the lower estimate of $F$-energy, we need the following lemma.

Lemma 3.1. Let $u:\left(M^{m}, f^{2} g_{0}\right) \rightarrow\left(N^{n}, h\right)$ be a $C^{2} F$-harmonic map with unique continuation property. Suppose $f$ satisfies $\left(\widetilde{f}_{1}\right)$. If $u$ is not a constant map, then the F-energy $E_{F}(u)$ must be infinite.

Proof. Using coarea formula, we have

$$
\begin{aligned}
\int_{M} F\left(\frac{|d u|^{2}}{2}\right) d v_{g} & =\int_{0}^{+\infty} \frac{d r}{r} r \int_{\partial B(r)} F\left(\frac{|d u|^{2}}{2}\right) \frac{1}{|\nabla r|} d s_{g} \\
& =\int_{0}^{+\infty} \frac{d r}{r} r \int_{\partial B(r)} F\left(\frac{|d u|^{2}}{2}\right) f d s_{g}
\end{aligned}
$$

where $B(r)$ is the geodesic ball centered at $x_{0}$ with radius $r$.

If $u$ has finite $F$-energy, i.e. $\int_{M} F\left(\frac{|d u|^{2}}{2}\right) d v_{g}<+\infty$, then by (3.15), we have a sequence $\left\{r_{i}\right\}$ such that

$$
\lim _{r_{i} \rightarrow+\infty} r_{i} \int_{\partial B\left(r_{i}\right)} F\left(\frac{|d u|^{2}}{2}\right) f d s_{g}=0 .
$$

By $\left(\widetilde{f}_{1}\right)$, the inequality (3.9) holds for $r(x) \geq R_{0}$. Using (3.8), we have

$$
r \frac{d}{d r} \int_{B(r)} F\left(\frac{|d u|^{2}}{2}\right) d v_{g} \geq \sigma \int_{B(r) \backslash B\left(R_{0}\right)} F\left(\frac{|d u|^{2}}{2}\right) d v_{g} .
$$

Let $r=r_{i}$ tend to infinity in the above inequality, using (3.16), we have $|d u|^{2}=0$ on $M^{m} \backslash B\left(R_{0}\right)$, that is, $u$ is constant on $M^{m} \backslash B\left(R_{0}\right)$. By the unique continuation property, we deduce that $u$ is constant on $M^{m}$. This contradiction shows that the $F$-energy $E_{F}(u)$ must be infinite.

By the above Lemma 3.1, we have

$$
\lim _{R \rightarrow \infty} \int_{B(R) \backslash B\left(R_{0}\right)} F\left(\frac{|d u|^{2}}{2}\right) d v_{g}=+\infty,
$$

and thus

$$
\int_{B(R) \backslash B\left(R_{0}\right)} F\left(\frac{|d u|^{2}}{2}\right) d v_{g}+\frac{H\left(R_{0}\right)}{\sigma}>0
$$

for sufficiently large $R$. Using (3.14), it follows that

$$
\frac{d}{d R}\left\{\frac{\int_{B(R) \backslash B\left(R_{0}\right)} F\left(\frac{|d u|^{2}}{2}\right) d v_{g}+\frac{H\left(R_{0}\right)}{\sigma}}{R^{\sigma}}\right\}>0
$$


which implies that

$$
\int_{B(R)} F\left(\frac{|d u|^{2}}{2}\right) d v_{g}+\frac{H\left(R_{0}\right)}{\sigma} \geq c(u) R^{\sigma}
$$

for sufficiently large $R$, where $c(u)>0$ is a constant only depending on $u$. Therefore we have the following proposition:

Proposition 3.2. Let $u:\left(M^{m}, f^{2} g_{0}\right) \rightarrow(N, h)$ be a $C^{2} F$-harmonic map with unique continuation property. Suppose $f$ satisfies $\left(\widetilde{f}_{1}\right)$. If $u$ is not a constant map, then we have the following estimate

$$
\int_{B(R)} F\left(\frac{|d u|^{2}}{2}\right) d v_{g} \geq c(u) R^{\sigma} \quad \text { as } R \rightarrow \infty
$$

where $c(u)>0$ is a constant only depending on $u$.

\section{Upper energy growth rates for $F$-harmonic maps}

In order to get the Liouville type property of $F$-harmonic maps, we need to estimate the upper $F$-energy of the $F$-harmonic maps.

Set

$$
E_{F}^{R}(u)=\int_{B(R)} F\left(\frac{|d u|^{2}}{2}\right) d v_{g} .
$$

Using a method similar to [Ji], we can derive the following theorem of an upper bound for the growth rate of $E_{F}^{R}(u)$ as $R \rightarrow \infty$.

Proposition 4.1. Let $u:\left(M^{m}, f^{2} g_{0}\right) \rightarrow\left(N^{n}, h\right)$ be a $C^{2}$ F-harmonic map. Suppose that $f$ satisfies $\left(f_{1}\right)$ and $\left(f_{2}\right)$, and the $F$-lower degree $l_{F}>0$ and $F^{\prime}\left(\frac{|d u|^{2}}{2}\right)<+\infty$. If $u(x) \rightarrow p_{o} \in N^{n}$ as $r(x) \rightarrow \infty$, then $u$ must be a constant map, or there exists constants $R_{0}, c(u)$, and $\eta(R) \rightarrow 0$ as $R \rightarrow \infty$, such that

$$
E_{F}^{R}(u) \leq C\left(\frac{\eta(R)}{2 l_{F}}+\frac{c(u)}{R^{\sigma}}\right) R^{\sigma} \quad \text { for } \quad R \geq R_{0} .
$$

Proof. Suppose the $F$-harmonic map is not constant, then by Proposition 3.1, the $F$-energy of $u$ must be infinite. That is, $E_{F}^{R}(u) \rightarrow \infty$ as $R \rightarrow \infty$.

Choose a local coordinate neighbourhood $(U, \varphi)$ of $p_{0}$ in $N^{n}$, such that $\varphi\left(p_{0}\right)=0$, it is clear that we can choose the $U$ in such a way that

$$
h=h_{\alpha \beta}(y) d y^{\alpha} \otimes d y^{\beta}, \quad y \in U
$$

satisfies

$$
\left(\frac{\partial h_{\alpha \beta(y)}}{\partial y^{\gamma}} y^{\gamma}+2 h_{\alpha \beta}(y)\right) \geq\left(h_{\alpha \beta}(y)\right) \quad \text { on } U
$$


in the matrices sense (that is, for two $n \times n$ matrices $A, B$, by $A \geq B$, we mean that $A-B$ is a positive semi-definite matrix).

Now the assumption that $u(x) \rightarrow 0$ as $r(x) \rightarrow \infty$ implies that there is an $R_{1}$ such that for $r(x)>R_{1}, u(x) \in U$, and

$$
\left(\frac{\partial h_{\alpha \beta(u)}}{\partial u^{\gamma}} y^{\gamma}+2 h_{\alpha \beta}(u)\right) \geq\left(h_{\alpha \beta}(u)\right) \quad \text { for } r(x)>R_{1} \text {. }
$$

For $w \in C_{0}^{2}\left(M^{m} \backslash B\left(R_{1}\right), \varphi(U)\right)$, we consider the variation $u+t w: M^{m} \rightarrow N^{n}$ defined as follows:

$$
(u+t w)(q)= \begin{cases}u(q) & \text { if } q \in B\left(R_{1}\right) \\ \varphi^{-1}[(\varphi(u)+t w)(q)] & \text { if } q \in M^{m} \backslash B\left(R_{1}\right)\end{cases}
$$

for sufficient small $t$. By the definition of $F$-harmonic maps, we have

$$
\left.\frac{d}{d t}\right|_{t=0} E_{F}(u+t w)=0
$$

that is,

$$
\int_{M^{m} \backslash B\left(R_{1}\right)} g_{0}^{i j} F^{\prime}\left(\frac{|d u|^{2}}{2}\right)\left[2 h_{\alpha \beta}(u) \frac{\partial u^{\alpha}}{\partial x_{i}} \frac{\partial w^{\beta}}{\partial x_{j}}+\frac{\partial h_{\alpha \beta}(u)}{\partial y^{\gamma}} w^{\gamma} \frac{\partial u^{\alpha}}{\partial x_{i}} \frac{\partial u^{\beta}}{\partial x_{j}}\right] f^{m-2}(x) d v_{g_{0}}=0 .
$$

Choose $w(x)=\phi(r(x)) u(x)$ in (4.2) for $\phi(t) \in C_{0}^{\infty}\left(R_{1}, \infty\right)$, we have

$$
\begin{aligned}
& \int_{M^{m} \backslash B\left(R_{1}\right)} g_{0}^{i j} F^{\prime}\left(\frac{|d u|^{2}}{2}\right)\left[2 h_{\alpha \beta}(u)+\frac{\partial h_{\alpha \beta}(u)}{\partial y^{\gamma}} u^{\gamma}\right] \frac{\partial u^{\alpha}}{\partial x_{i}} \frac{\partial u^{\beta}}{\partial x_{j}} \phi(r(x)) f^{m-2}(x) d v_{g_{0}} \\
= & -2 \int_{M^{m} \backslash B\left(R_{1}\right)} g_{0}^{i j} F^{\prime}\left(\frac{|d u|^{2}}{2}\right) h_{\alpha \beta}(u) \frac{\partial u^{\alpha}}{\partial x_{i}} u^{\beta} \frac{\partial \phi(r(x))}{\partial x_{j}} f^{m-2}(x) d v_{g_{0}} .
\end{aligned}
$$

By a standard approximation argument, (4.3) holds for Lipschitz function $\phi$ with compact support.

For $0<\epsilon \leq 1$, define

$$
\varphi_{\epsilon}(t)= \begin{cases}1 & t \leq 1 \\ 1+\frac{1-t}{\epsilon} & 1<t<1+\epsilon \\ 0 & t \geq 1+\epsilon .\end{cases}
$$

In (4.3), choose the Lipschitz function $\phi(r(x))$ to be

$$
\phi(r(x))=\varphi_{\epsilon}\left(\frac{r(x)}{R}\right)\left(1-\varphi_{1}\left(\frac{r(x)}{R_{1}}\right)\right), \quad R>2 R_{1} .
$$


Set $\nu^{i}=g_{0}^{i j} \frac{\partial r}{\partial x_{j}}$, and thus $\nu=\nu^{i} \frac{\partial}{\partial x_{i}}$ is the outer normal vector field along $\partial B(R)$. Let $\epsilon \rightarrow 0$, notice

$$
\frac{\partial \varphi_{\epsilon}\left(\frac{r(x)}{R}\right)}{\partial x_{i}}=-\frac{1}{R \epsilon} \frac{\partial r(x)}{\partial x_{i}} \text { for } R<r(x)<R(1+\epsilon)
$$

and

$$
\begin{aligned}
& \lim _{\epsilon \rightarrow 0} \frac{1}{R \epsilon} \int_{B(R(1+\epsilon)) \backslash B(R)} F^{\prime}\left(\frac{|d u|^{2}}{2}\right) h_{\alpha \beta}(u) \frac{\partial u^{\alpha}}{\partial x_{i}} \nu^{i} u^{\beta} f^{m-2}(x) d v_{g_{0}} \\
& =\int_{\partial B(R)} F^{\prime}\left(\frac{|d u|^{2}}{2}\right) h_{\alpha \beta}(u) \frac{\partial u^{\alpha}}{\partial x_{i}} \nu^{i} u^{\beta} f^{m-2}(x) d s_{g_{0}},
\end{aligned}
$$

we get $\left(R_{2}=2 R_{1}\right)$

$$
\begin{aligned}
& \int_{B(R) \backslash B\left(R_{2}\right)} g_{0}^{i j} F^{\prime}\left(\frac{|d u|^{2}}{2}\right)\left[2 h_{\alpha \beta}(u)+\frac{\partial h_{\alpha \beta}(u)}{\partial y^{\gamma}} u^{\gamma}\right] \frac{\partial u^{\alpha}}{\partial x_{i}} \frac{\partial u^{\beta}}{\partial x_{j}} f^{m-2}(x) d v_{g_{0}}+D\left(R_{1}\right) \\
= & 2 \int_{\partial B(R)} F^{\prime}\left(\frac{|d u|^{2}}{2}\right) h_{\alpha \beta}(u) \frac{\partial u^{\alpha}}{\partial x_{i}} \nu^{i} u^{\beta} f^{m-2}(x) d s_{g_{0}}
\end{aligned}
$$

where

$$
\begin{aligned}
D\left(R_{1}\right)= & \int_{B\left(R_{2}\right) \backslash B\left(R_{1}\right)} g_{0}^{i j} F^{\prime}\left(\frac{|d u|^{2}}{2}\right)\left\{\left[\left(2 h_{\alpha \beta}(u)+\frac{\partial h_{\alpha \beta}(u)}{\partial y^{\gamma}} u^{\gamma}\right) \frac{\partial u^{\alpha}}{\partial x_{i}} \frac{\partial u^{\beta}}{\partial x_{j}}\left(1-\varphi_{1}\left(\frac{r(x)}{R_{1}}\right)\right)\right]\right. \\
& \left.-2 h_{\alpha \beta}(u) \frac{\partial u^{\alpha}}{\partial x_{i}} u^{\beta} \frac{\partial \varphi_{1}\left(\frac{r(x)}{R_{1}}\right)}{\partial x_{j}}\right\} f^{m-2}(x) d v_{g_{0}} .
\end{aligned}
$$

Set

$$
Z(R)=\int_{B(R) \backslash B\left(R_{2}\right)} g_{0}^{i j} F^{\prime}\left(\frac{|d u|^{2}}{2}\right) h_{\alpha \beta}(u) \frac{\partial u^{\alpha}}{\partial x_{i}} \frac{\partial u^{\beta}}{\partial x_{j}} f^{m-2}(x) d v_{g_{0}}+D\left(R_{1}\right) \text { for } \quad R>R_{2}
$$

then

$$
Z^{\prime}(R)=\int_{\partial B(R)} g_{0}^{i j} F^{\prime}\left(\frac{|d u|^{2}}{2}\right) h_{\alpha \beta}(u) \frac{\partial u^{\alpha}}{\partial x_{i}} \frac{\partial u^{\beta}}{\partial x_{j}} f^{m-2}(x) d s_{g_{0}}
$$

Notice that

$$
h_{\alpha \beta}(u) \frac{\partial u^{\alpha}}{\partial x_{i}} \nu^{i} u^{\beta}=\left\langle\frac{\partial u^{\alpha}}{\partial x_{i}} d x^{i} \otimes \frac{\partial}{\partial y^{\alpha}}, \frac{\partial r}{\partial x_{j}} u^{\beta} d x^{j} \otimes \frac{\partial}{\partial y^{\beta}}\right\rangle_{g_{0} \otimes h}
$$


and therefore

$$
\begin{aligned}
& \int_{\partial B(R)} F^{\prime}\left(\frac{|d u|^{2}}{2}\right) h_{\alpha \beta}(u) \frac{\partial u^{\alpha}}{\partial x_{i}} \nu^{i} u^{\beta} f^{m-2} d s_{g_{0}} \\
& =\int_{\partial B(R)}\left\langle\frac{\partial u^{\alpha}}{\partial x_{i}} d x^{i} \otimes \frac{\partial}{\partial y^{\alpha}}, \frac{\partial r}{\partial x_{j}} u^{\beta} d x^{j} \otimes \frac{\partial}{\partial y^{\beta}}\right\rangle_{g_{0} \otimes h} F^{\prime}\left(\frac{|d u|^{2}}{2}\right) f^{m-2} d s_{g_{0}} \\
& \leq \int_{\partial B(R)}\left|\frac{\partial u^{\alpha}}{\partial x_{i}} d x^{i} \otimes \frac{\partial}{\partial y^{\alpha}} \| \frac{\partial r}{\partial x_{j}} u^{\beta} d x^{j} \otimes \frac{\partial}{\partial y^{\beta}}\right| F^{\prime}\left(\frac{|d u|^{2}}{2}\right) f^{m-2} d s_{g_{0}} \\
& \leq \sqrt{\int_{\partial B(R)}\left|\frac{\partial u^{\alpha}}{\partial x_{i}} d x^{i} \otimes \frac{\partial}{\partial y^{\alpha}}\right|_{g_{0} \otimes h}^{2} \mid F^{\prime}\left(\frac{|d u|^{2}}{2}\right) f^{m-2} d s_{g_{0}}} \\
& \times \sqrt{\int_{\partial B(R)}\left|\frac{\partial r}{\partial x_{j}} u^{\beta} d x^{j} \otimes \frac{\partial}{\partial y^{\beta}}\right|_{g_{0} \otimes h}^{2} \mid F^{\prime}\left(\frac{|d u|^{2}}{2}\right) f^{m-2} d s_{g_{0}}} \\
& =\sqrt{\int_{\partial B(R)} g_{0}^{i j} h_{\alpha \beta}(u) \frac{\partial u^{\alpha}}{\partial x_{i}} \frac{\partial u^{\beta}}{\partial x_{j}} F^{\prime}\left(\frac{|d u|^{2}}{2}\right) f^{m-2} d s_{g_{0}}} \\
& \times \sqrt{\int_{\partial B(R)} g_{0}^{i j} h_{\alpha \beta}(u) \frac{\partial r}{\partial x_{i}} \frac{\partial r}{\partial x_{j}} u^{\alpha} u^{\beta} F^{\prime}\left(\frac{|d u|^{2}}{2}\right) f^{m-2} d s_{g_{0}}} \\
& =\sqrt{\int_{\partial B(R)} g_{0}^{i j} h_{\alpha \beta}(u) \frac{\partial u^{\alpha}}{\partial x_{i}} \frac{\partial u^{\beta}}{\partial x_{j}} F^{\prime}\left(\frac{|d u|^{2}}{2}\right) f^{m-2} d s_{g_{0}}} \\
& \times \sqrt{\int_{\partial B(R)} h_{\alpha \beta}(u) u^{\alpha} u^{\beta} F^{\prime}\left(\frac{|d u|^{2}}{2}\right) f^{m-2} d s_{g_{0}}},
\end{aligned}
$$

where the last equality is because of

$$
g_{0}^{i j} \frac{\partial r}{\partial x_{i}} \frac{\partial r}{\partial x_{j}}=\left\langle\nabla^{0} r, \nabla^{0} r\right\rangle_{g_{0}}=1
$$

By the definition of $l_{F}$, we have

$$
\int_{B(R) \backslash B\left(R_{2}\right)} g_{0}^{i j} F^{\prime}\left(\frac{|d u|^{2}}{2}\right) h_{\alpha \beta}(u) \frac{\partial u^{\alpha}}{\partial x_{i}} \frac{\partial u^{\beta}}{\partial x_{j}} f^{m-2}(x) d v_{g_{0}} \geq 2 l_{F} \int_{B(R) \backslash B\left(R_{2}\right)} F\left(\frac{|d u|^{2}}{2}\right) d v_{g} .
$$

Since $l_{F}>0$ and $E_{F}^{R} \rightarrow \infty$ as $R \rightarrow \infty$, there is an $R_{3} \geq R_{2}$, such that $Z(R)>0$ for $R \geq R_{3}$. Thus (4.1), (4.4) and (4.5) imply

$$
Z(R)^{2} \leq C Z^{\prime}(R)\left(\int_{\partial B(R)} F^{\prime}\left(\frac{|d u|^{2}}{2}\right) h_{\alpha \beta}(u) u^{\alpha} u^{\beta} f^{m-2}(x) d s_{g_{0}}\right) \quad \text { for } \quad R>R_{3} .
$$

If we denote

$$
M(R)=\int_{\partial B(R)} F^{\prime}\left(\frac{|d u|^{2}}{2}\right) h_{\alpha \beta}(u) u^{\alpha} u^{\beta} f^{m-2}(x) d s_{g_{0}}
$$


then for $R_{4} \geq R \geq R_{3}$, it follows that

$$
\int_{R}^{R_{4}}\left(\frac{-1}{Z(r)}\right)^{\prime} d r \geq C \int_{R}^{R_{4}} \frac{1}{M(r)} d r
$$

Let $R_{4} \rightarrow \infty$ and notice that $Z(R)>0$, we have

$$
\frac{1}{Z(R)} \geq C \int_{R}^{\infty} \frac{1}{M(r)} d r
$$

Thus

$$
Z(R) \leq C \frac{1}{\int_{R}^{\infty} \frac{1}{M(r)} d r} \text { for } R>R_{3}
$$

By $F^{\prime}\left(\frac{|d u|^{2}}{2}\right)<+\infty$ and the fact that $u(x) \rightarrow 0$ as $r(x) \rightarrow \infty$, we get

$$
M(R) \leq C \eta(R) \int_{\partial B(R)} f^{m-2}(x) d s_{g_{0}}
$$

where $\eta(R)$ is chosen in such a way that

(i) $\eta(R)$ is nonincreasing on $\left(R_{3}, \infty\right)$ and $\eta(R) \rightarrow 0$ as $R \rightarrow \infty$;

(ii) $\eta(R) \geq \max _{r(x)=R}\left\{h_{\alpha \beta}(u) u^{\alpha} u^{\beta}\right\}$.

Then by $\left(f_{2}\right)$, we derive

$$
\int_{R}^{\infty} \frac{1}{M(r)} d r \geq \frac{C}{\eta(R)} \int_{R}^{\infty} \frac{1}{\int_{\partial B(r)} f^{m-2}(x) d s_{g_{0}}} d r \geq \frac{C}{\eta(R)} R^{-\sigma}
$$

Thus

$$
Z(R) \leq C \eta(R) R^{\sigma} \quad \text { for } R \geq R_{3} .
$$

Therefore, using (4.6), we obtain

$$
E_{F}^{R}(u) \leq C\left(\frac{\eta(R)}{2 l_{F}}+\frac{c(u)}{R^{\sigma}}\right) R^{\sigma}
$$

Remark 4.1. When the F-harmonic map $u$ has the unique continuation property, then by Lemma 3.1, the conclusion of Proposition 4.1 also holds for $u$ with the condition $\left(f_{1}\right)$ replaced by $\left(\tilde{f}_{1}\right)$.

\section{The main results and their proof}

Combining Proposition 3.1 and Proposition 4.1, we have the following Liouville type theorem. 
Theorem 5.1. Let $u:\left(M^{m}, f^{2} g_{0}\right) \rightarrow\left(N^{n}, h\right)$ be a $C^{2} F$-harmonic map. Suppose that $f$ satisfies $\left(f_{1}\right)$ and $\left(f_{2}\right)$, and that the $F$-lower degree $l_{F}>0$ and $F^{\prime}\left(\frac{|d u|^{2}}{2}\right)<+\infty$. If $u(x) \rightarrow p_{o} \in N^{n}$ as $r(x) \rightarrow \infty$, then $u$ is a constant map.

Remark 5.1. Assuming either $\sup _{t \geq 0} F^{\prime}(t)<+\infty$ or $\sup _{M}|d u|^{2}<+\infty$, we may deduce that $F^{\prime}\left(\frac{|d u|^{2}}{2}\right)<C$.

By Proposition 3.2 and Remark 4.1, we have the following theorem for $F$-harmonic map with the unique continuation property, which includes the case of harmonic maps in Jin's paper ([Ji]).

Theorem 5.2. Let $u:\left(M^{m}, f^{2} g_{0}\right) \rightarrow\left(N^{n}, h\right)$ be a $C^{2}$ F-harmonic map with the unique continuation property. Suppose that $f$ satisfies $\left(\tilde{f}_{1}\right)$ and $\left(f_{2}\right)$, and that the $F$-lower degree $l_{F}>0$ and $F^{\prime}\left(\frac{|d u|^{2}}{2}\right)<+\infty$. If $u(x) \rightarrow p_{o} \in N^{n}$ as $r(x) \rightarrow \infty$, then $u$ is a constant map.

Corollary 5.1. Let $u:\left(M^{m}, g_{0}\right) \rightarrow\left(N^{n}, h\right)$ be a $C^{2} F$-harmonic map. There are positive constants $C, \sigma$ and $R_{0}$ such that

$$
\begin{aligned}
& \frac{(m-1)}{2} \lambda_{\min }+1-d_{F} \max \left\{2, \lambda_{\max }\right\} \geq \sigma \\
& \left(\int_{R}^{\infty} \frac{d r}{\operatorname{vol}(\partial B(r))}\right)^{-1} \leq C R^{\sigma} \quad \text { for } \quad R>R_{0} .
\end{aligned}
$$

The lower degree $l_{F}>0$ and $F^{\prime}\left(\frac{|d u|^{2}}{2}\right)<+\infty$. If $u(x) \rightarrow p_{o} \in N^{n}$ as $r(x) \rightarrow \infty$, then $u$ is a constant map.

When applied the above results to some concrete pinched manifolds, we need the following lemmas.

Lemma 5.1. Let $\left(M, g_{0}\right)$ be an m-dimensional complete Riemannian manifold with a pole $x_{0}$ and let $r(x)$ be the distance function relative to $x_{0}$. Assume that there exist two positive functions $h_{1}(r)$ and $h_{2}(r)$ such that

$$
h_{1}(r)\left[g_{0}-d r \otimes d r\right] \leq \operatorname{Hess}(r) \leq h_{2}(r)\left[g_{0}-d r \otimes d r\right]
$$

in the sense of quadratic forms, then

$$
\frac{(m-1)}{2} \lambda_{\min }+1-d_{F} \max \left\{2, \lambda_{\max }\right\} \geq(m-1) h_{1}(r) r+1-2 d_{F} \max \left\{1, h_{2}(r) r\right\} .
$$

Proof. Applying the Hessian operator to the composed function $r^{2}$, we have

$$
\operatorname{Hess}\left(r^{2}\right)=2 r \operatorname{Hess}(r)+2 d r \otimes d r
$$

which immediately yields the result. 
Lemma 5.2. (cf. $[G W],[D W],[P R S])$ Let $\left(M, g_{0}\right)$ be a complete Riemannian manifold with a pole $x_{0}$ and let $r$ be the distance function relative to $x_{0}$. Denote by $K_{r}$ the radial curvature of $M$.

(i) If $-\alpha^{2} \leq K_{r} \leq-\beta^{2}$ with $\alpha>0, \beta>0$, then

$$
\beta \operatorname{coth}(\beta r)[g-d r \otimes d r] \leq H e s s(r) \leq \alpha \operatorname{coth}(\alpha r)[g-d r \otimes d r] .
$$

(ii) If $-\frac{A}{\left(1+r^{2}\right)^{1+\epsilon}} \leq K_{r} \leq \frac{B}{\left(1+r^{2}\right)^{1+\epsilon}}$ with $\epsilon>0, A \geq 0,0 \leq B<2 \epsilon$, then

$$
\frac{1-\frac{B}{2 \epsilon}}{r}[g-d r \otimes d r] \leq \operatorname{Hess}(r) \leq \frac{e^{\frac{A}{2 \epsilon}}}{r}[g-d r \otimes d r]
$$

(iii) If $-\frac{a^{2}}{1+r^{2}} \leq K_{r} \leq \frac{b^{2}}{1+r^{2}}$ with $a \geq 0, b^{2} \in[0,1 / 4]$, then

$$
\frac{1+\sqrt{1-4 b^{2}}}{2 r}[g-d r \otimes d r] \leq \operatorname{Hess}(r) \leq \frac{1+\sqrt{1+4 a^{2}}}{2 r}[g-d r \otimes d r] .
$$

By Theorem 5.1, Lemma 5.1 and Lemma 5.2, we can get the following theorem.

Theorem 5.3. Let $u:\left(M^{m}, f^{2} g_{0}\right) \rightarrow\left(N^{n}, h\right)$ be a $C^{2} F$-harmonic map. Suppose $f$ satisfies $\frac{\partial \log f}{\partial r} \geq 0$ and there exist a constant $\sigma>0$ such that the inequality in $\left(f_{2}\right)$ holds. The F-lower degree $l_{F}>0$ and $F^{\prime}\left(\frac{|d u|^{2}}{2}\right)<+\infty$. Suppose $M^{m}$ is a complete Riemannian manifold with a pole and its radial curvature satisfies one of the following three conditions:

(i) $-\alpha^{2} \leq K_{r} \leq-\beta^{2}$ with $\alpha>0, \beta>0$ and $r \frac{\partial \log f}{\partial r}\left(m-2 d_{F}\right)+1+(m-$ 1) $\beta r \operatorname{coth}(\beta r)-2 d_{F} \alpha r \operatorname{coth}(\alpha r) \geq \sigma$;

(ii) $-\frac{A}{\left(1+r^{2}\right)^{1+\epsilon}} \leq K_{r} \leq \frac{B}{\left(1+r^{2}\right)^{1+\epsilon}}$ with $\epsilon>0, A \geq 0,0 \leq B<2 \epsilon$ and $r \frac{\partial \log f}{\partial r}(m-$ $\left.2 d_{F}\right)+1+(m-1)\left(1-\frac{B}{2 \epsilon}\right)-2 d_{F} e^{\frac{A}{2 \epsilon}} \geq \sigma$

(iii) $-\frac{a^{2}}{1+r^{2}} \leq K_{r} \leq \frac{b^{2}}{1+r^{2}}$ with $a \geq 0, b^{2} \in[0,1 / 4]$ and $r \frac{\partial \log f}{\partial r}\left(m-2 d_{F}\right)+1+(m-$ 1) $\frac{1+\sqrt{1-4 b^{2}}}{2}-d_{F}\left(1+\sqrt{1+4 a^{2}}\right) \geq \sigma$.

If $u(x) \rightarrow p_{o} \in N^{n}$ as $r(x) \rightarrow \infty$, then $u$ is a constant map.

Remark 5.2. (i) If $(m-1) \beta-2 d_{F} \alpha \geq 0$, then we have

$$
\begin{aligned}
(m-1) \beta r \operatorname{coth}(\beta r)-2 d_{F} \alpha r \operatorname{coth}(\alpha r) & \geq \beta r \operatorname{coth}(\beta r)\left[(m-1)-2 d_{F} \frac{\alpha r \operatorname{coth}(\alpha r)}{\beta r \operatorname{coth}(\beta r)}\right] \\
& \geq(m-1)-2 d_{F} \frac{\alpha}{\beta}
\end{aligned}
$$

since $\beta r \operatorname{coth}(\beta r)>1$ for $r>0$, and $\frac{\alpha r \operatorname{coth}(\alpha r)}{\beta r \operatorname{coth}(\beta r)}<1$ for $0<\beta<\alpha$, and coth is a decreasing function. Thus the conclusion of the first case in Theorem 5.3 still holds if $r \frac{\partial \log f}{\partial r}\left(m-2 d_{F}\right)+m-2 d_{F} \frac{\alpha}{\beta} \geq \sigma$.

(ii) If $f$ satisfies $\frac{\partial \log f}{\partial r} \leq 0$ and the condition $\left(f_{2}\right)$, then the conclusion in Theorem 5.3 still holds provided that one replaces $m-2 d_{F}$ by $m-2 l_{F}$ in (i) (ii) (iii).

(iii) M. Kassi ([Ka]) proved a Liouville theorem for F-harmonic maps from various pinched manifolds, which has finite F-energy and some restrictions on $d_{F}$. 
Taking $f=1$, we have the following corollary.

Corollary 5.2. Let $u:\left(M^{m}, g_{0}\right) \rightarrow\left(N^{n}, h\right)$ be a $C^{2} F$-harmonic map. The F-lower degree $l_{F}>0$ and $F^{\prime}\left(\frac{|d u|^{2}}{2}\right)<+\infty$. Suppose $M^{m}$ is a complete Riemannian manifold with a pole and its radial curvature satisfies one of the following two conditions:

(i) $-\frac{A}{\left(1+r^{2}\right)^{1+\epsilon}} \leq K_{r} \leq \frac{B}{\left(1+r^{2}\right)^{1+\epsilon}}$ with $\epsilon>0, A \geq 0,0 \leq B<2 \epsilon$ and $1+(m-1)(1-$ $\left.\frac{B}{2 \epsilon}\right)-2 d_{F} e^{\frac{A}{2 \epsilon}} \geq m-2$;

(ii) $-\frac{a^{2}}{1+r^{2}} \leq K_{r} \leq \frac{b^{2}}{1+r^{2}}$ with $a \geq 0, b^{2} \in[0,1 / 4]$ and $1+(m-1) \frac{1+\sqrt{1-4 b^{2}}}{2}-d_{F}(1+$ $\left.\sqrt{1+4 a^{2}}\right) \geq(m-1) A^{\prime}-1$, where $A^{\prime}=\frac{1+\sqrt{1+4 a^{2}}}{2}$.

If $u(x) \rightarrow p_{o} \in N^{n}$ as $r(x) \rightarrow \infty$, then $u$ is a constant map.

Proof. For the first case (i), it follows that

$$
\operatorname{Ric}_{g_{0}}(x) \geq-\frac{(m-1) A}{\left(1+r^{2}(x)\right)^{1+\epsilon}} \quad \forall x \in M^{m} .
$$

By direct calculation

$$
\int_{0}^{\infty} \frac{A r}{\left(1+r^{2}\right)^{1+\epsilon}} d r=\frac{A}{2 \epsilon}
$$

Thus using the volume comparison theorem (cf. [PRS]), we have

$$
\operatorname{vol}_{g_{0}}(\partial B(R)) \leq \omega_{m} e^{\frac{(m-1) A}{2 \epsilon}} R^{m-1}
$$

where $\omega_{m}$ is the $(m-1)$-volume of the unit sphere in $R^{m}$, and thus

$$
\left(\int_{R}^{\infty} \frac{d r}{\operatorname{vol}_{g_{0}}(\partial B(r))}\right)^{-1} \leq(m-2) \omega_{m} e^{\frac{(m-1) A}{2 \epsilon}} R^{m-2} \quad \text { for } \quad R>R_{0} .
$$

For the second case (ii), it follows that

$$
\operatorname{Ric}_{g_{0}}(x) \geq-\frac{(m-1) a^{2}}{1+r^{2}(x)} \forall x \in M^{m} .
$$

Then the volume comparison theorem yields (cf. [PRS])

$$
\operatorname{vol}_{g_{0}}(\partial B(R)) \leq C R^{(m-1) A^{\prime}}
$$

where $A^{\prime}=\frac{1+\sqrt{1+4 a^{2}}}{2}$. Thus

$$
\left(\int_{R}^{\infty} \frac{d r}{\operatorname{vol}_{g_{0}}(\partial B(r))}\right)^{-1} \leq C R^{(m-1) A^{\prime}-1} \text { for } R>R_{0} .
$$

Therefore, using Corollary 5.1, the conclusion of this corollary is immediately proved.

Remark 5.3. If $-\alpha^{2} \leq K_{r} \leq-\beta^{2}$ with $\alpha>0, \beta>0$, then the volume of $\partial B(R)$ has exponential growth, thus the condition (5.2) doesn't hold for any $\sigma>0$, so we needn't consider the case (i) in Theorem 5.3. 
Corollary 5.3. Let $u:\left(R^{m}, g_{0}\right) \rightarrow\left(N^{n}, h\right)$ be a $C^{2} F$-harmonic map. Suppose the $F$-lower degree $l_{F}>0$ and $F^{\prime}\left(\frac{|d u|^{2}}{2}\right)<+\infty$. If $d_{F} \leq 1$ and $u(x) \rightarrow p_{0} \in N^{n}$ as $|x| \rightarrow \infty$, then $u$ is a constant map.

Proof. Consider the case $A=B=0$ in Corollary 5.2 (i). If $d_{F} \leq 1$, then the conditions in that corollary are satisfied by choosing $\sigma=m-2$, and the conclusion of this corollary follows immediately.

Remark 5.4. For the harmonic map $u$, it is an F-harmonic map with $F=2 t$, $d_{F}=l_{F}=1, F^{\prime}(t)=2$. Thus Theorem $A$ of [Ji] stated in the introduction can be regarded as one version of this corollary in the case of harmonic maps.

Theorem 5.4. Suppose $f$ satisfies $\left(f_{1}\right)$ and $\left(f_{3}\right)$ and the $F$-lower degree $l_{F}>0$. Then for any $p \in N^{n}$, there is an (nonempty) open neighbourhood $U_{p} \subset N^{n}$, such that the family of open sets $\left\{U_{p} \mid p \in N^{n}\right\}$ has following property:

If $u:\left(M^{m}, f^{2} g_{0}\right) \rightarrow\left(N^{n}, h\right)$ is a $C^{2}$ harmonic map, $F^{\prime}\left(\frac{|d u|^{2}}{2}\right)<+\infty$, and for some $p \in N^{n}, u(x) \in U_{p}$ as $r(x) \rightarrow \infty$, then $u$ is a constant map.

Proof. The proof of Theorem 5.4 is a modification of the proof of Theorem 5.1. Choose a family of coordinate neighbourhoods $\left\{U_{p} \mid p \in N^{n}\right\}$ as follows: let $\left(U_{p}, \varphi\right)$ be a coordinate system centered at $p$ such that

$$
\left(\frac{\partial h_{\alpha \beta(y)}}{\partial y^{\gamma}} y^{\gamma}+2 h_{\alpha \beta}(y)\right) \geq\left(h_{\alpha \beta}(y)\right) \quad \text { on } U_{p}
$$

and

$$
h_{\alpha \beta}(y) y^{\alpha} y^{\beta} \leq C_{p}
$$

where $C_{p}$ is an arbitrary constant which may depend on $p$. Then we claim that this family $\left\{U_{p} \mid p \in N^{n}\right\}$ is what we want.

In fact, if $u:\left(M^{m}, f^{2} g_{0}\right) \rightarrow\left(N^{n}, h\right)$ is a non-constant $C^{2}$ harmonic map, and for some $p \in N^{n}, u(x) \in U_{p}$ as $r(x) \rightarrow \infty$, then we may assume that for some $R_{0}$, $u(x) \in U_{p}$ for $r(x)>R_{0}$. Proceeding as in the proof of Theorem 5.1, we get

$$
\frac{1}{Z(R)} \geq C \int_{R}^{\infty} \frac{1}{M(r)} d r, \text { for } R>R_{3} .
$$

But in this case

$$
M(R) \leq C C_{p} \int_{\partial B(R)} f^{m-2}(x) d s_{g_{0}} \leq C C_{p} R \log R .
$$

Therefore

$$
\int_{R}^{\infty} \frac{1}{M(r)} d r \geq \frac{1}{C C_{p}} \int_{R}^{\infty} \frac{1}{r \log R} d r=\infty .
$$

Now we have a contradiction to (5.3), since if $\mathrm{u}$ is not a constant map, $Z(R)>0$ for $R$ large.

It is interesting to note that different $F(t)$ may have the same upper degree or the lower degree. Therefore the results in this section may be applied simultaneously to different $F$-harmonic maps. 


\section{A further theorem and its application}

In this section, we show that the asymptotic condition on $F$-harmonic maps for Liouville theorems can be relaxed if the target manifold is more special. Let $\left(R^{n}, h_{0}\right)$ be the standard Euclidean space, where $h_{0}=\sum_{\alpha=1}^{n}\left(d y_{\alpha}\right)^{2}$. Denote by $\rho(y)=\operatorname{dist}_{h_{0}}(y, o)$ the standard Euclidean distance relative to the origin. If we choose a function $\lambda(\rho)=$ $k_{1} \rho^{k-1}$, where $k_{1}>0$ and $k \geq 1$, then it is easy to verify that the metric $h(y)=$ $\lambda^{2}(\rho(y)) h_{0}(y)$ satisfies

$$
\left(\frac{\partial h_{\alpha \beta(y)}}{\partial y^{\gamma}} y^{\gamma}+2 h_{\alpha \beta}(y)\right) \geq\left(h_{\alpha \beta}(y)\right)
$$

Let $u:\left(M^{m}, g\right) \rightarrow\left(R^{n}, h\right)$ be a $F$-harmonic map. Notice that the whole image of $u$ is contained in a global coordinate of $R^{n}$. So one may construct the variation $u+t w$ as in $\S 4$. Therefore all integral formulae and inequalities in $\S 4$ still hold just by taking $R_{1}=0$.

Set

$$
K(R)=\max _{r(x)=R}\left\{F^{\prime}\left(\frac{|d u|^{2}}{2}\right) h_{\alpha \beta}(u) u^{\alpha} u^{\beta}\right\} .
$$

By using a small modified method of Theorem 5.1, we can get the following theorem.

Theorem 6.1. Let $u:\left(M^{m}, f^{2} g_{0}\right) \rightarrow\left(R^{n}, h\right)$ be a $C^{2} F$-harmonic map. Suppose that $f$ satisfies $\left(f_{1}\right)$ and $\left(f_{2}\right)$ and the $F$-lower degree $l_{F}>0$. If $\lim _{R \rightarrow \infty} K(R)=0$, then $u$ is a constant map.

Proof. By (4.7), we have

$$
M(R) \leq K(R) \int_{\partial B(R)} f^{m-2}(x) d s_{g_{0}} .
$$

Since $K(R)$ is continuous and $\lim _{R \rightarrow \infty} K(R)=0$, we can choose a function $\bar{K}(R)$ such that

(i) $\bar{K}(R) \geq K(R)$;

(ii) $\bar{K}(R)$ is nonincreasing on $\left(R_{3}, \infty\right)$ and $\bar{K}(R) \rightarrow 0$ as $R \rightarrow \infty$, where $R_{3}$ is the constant in $\S 4$.

Thus the remaining part of the proof is similar to that of Theorem 5.1. We omit the details.

It follows immediately that

Corollary 6.1. Let $u:\left(M^{m}, f^{2} g_{0}\right) \rightarrow R$ be a $C^{2} F$-harmonic function. Suppose that $f$ satisfies $\left(f_{1}\right)$ and $\left(f_{2}\right)$, and the $F$-lower degree $l_{F}>0$. If

$$
\left.\lim _{R \rightarrow \infty r(x)=R} \max _{2}\left\{\frac{|d u|^{2}}{2}\right)|u|^{2}\right\}=0
$$

then $u$ is a constant map. 
Corollary 6.2. Let $x_{m+1}=u\left(x_{1}, \cdots, x_{m}\right)$ be an entire minimal graph on $R^{m}(m>$ $2)$, where $x=\left(x_{1}, \cdots, x_{m}\right) \in R^{m}$ is the standard Euclidean coordinate. If

$$
\lim _{R \rightarrow \infty|x|=R} \max _{2}\left\{\frac{(u-c)^{2}}{\sqrt{1+|d u|^{2}}}\right\}=0,
$$

then the graph is a horizontal hyperplane.

Proof. From Example 2.2, we know that $u:\left(R^{m}, g_{0}\right) \rightarrow R$ is a $F$-harmonic map with $F(t)=\sqrt{1+2 t}-1$.

By a direct calculation, we have $l_{F}=\frac{1}{2}, d_{F}=1, F^{\prime}\left(\frac{|d u|^{2}}{2}\right)=\frac{1}{\sqrt{1+|d u|^{2}}}<+\infty$, and $\lambda_{\min }=\lambda_{\max }=2$. Thus

$$
\frac{(m-1)}{2} \lambda_{\min }+1-d_{F} \max \left\{2, \lambda_{\max }\right\}=m-2
$$

and

$$
\left(\int_{R}^{\infty} \frac{d r}{\operatorname{vol}(\partial B(r))}\right)^{-1}=m(m-2) \omega_{m} R^{m-2}
$$

where $\omega_{m}$ is the volume of the unit sphere in $R^{m}$. Clearly the minimality of the graph is invariant under the upward or downward movement in $x_{m+1}$-axis direction. Thus the conditions of Corollary 6.1 are satisfied if we choose $C=m(m-2) \omega_{m}$ and $\sigma=m-2$, therefore $u$ is constant and the graph is a horizontal hyperplane.

Remark 6.1. (i) When $m \leq 7$, it is well known that the entire graph $x_{m+1}=$ $u\left(x_{1}, \cdots, x_{m}\right)$ over $R^{m}$ is a hyperplane in $R^{m+1}$ (cf. [Si]). We know that $\frac{1}{\sqrt{1+|d u|^{2}}}=$ $\cos (\theta)$, where $\theta$ is the angle function between the normal vector field of the graph and the $x_{m+1}$-axis. Actually we may state Corollary 6.2 in a more geometric way: Let $P$ be an m-dimensional hyperplane in $R^{m+1}$. Suppose $M^{m}$ is a complete minimal hypersurface in $R^{m+1}$ which is a graph over the plane $P$. Denote by $\theta$ the angle function between the normal vector field of $M$ and the normal vector of $P$. For $q \in M$, let $d(q, P)$ be the Euclidean distance from $q$ to $P$. If

$$
\lim _{q \rightarrow \infty} d^{2}(q, P) \cos \theta(q)=0
$$

then $M$ is a hyperplane parallel to $P$.

(ii) From Corollary 6.2, we may deduce the following result: If there exists a constant $c$ such that $\lim _{|x| \rightarrow \infty} u(x)=c$, then $u=c$. Note that the later result is also a consequence of Simon's result (cf. Lemma 1.1 in [Sim]).

Acknowledgements. The authors would like to thank Prof. S.S. Wei and Prof. T.H. Otway for their valuable comments and suggestions. They would also like to thank Dr. Qingchun Ji and Dr. Y.B. Ren for their helpful discussions. 


\section{References}

[Ar] M. Ara, Geometry of F-harmonic maps, Kodai Math. 22 (1999), 243-263.

[Ba] P. Baird: Stress-energy tensors and the Lichnerowicz Laplacian, Journal of Geom. and Phys. 58 (2008), 1329-1342.

[Ch] S.Y. Cheng, Liouville theorem for harmonic maps, Pure Math., vol. 36, American Mathematical Society, Providence, R.T., (1980), 147-151.

[DW] Y.X. Dong, S.S. Wei, On vanishing theorems for vector bundle valued $p$-forms and their applications, Comm. Math. Phys. Vol. 304 (2011), 329-368.

[GRSB] W.D. Garber, S.N.M. Ruijsennaars, E. Seiler, D. Burns, On finite action solutions of the nonlinear $\sigma$-model, Ann. Phys. 119 (1979), 305-325.

[GW] R.E. Greene, H. Wu, Function theory on manifolds which posses a pole, Lecture Notes in Math. Vol.699, Springer-Verlag, 1979.

[Hi] S. Hildebrandt, Liouville theorems for harmonic mappings, and an approach to Bernstein theorems, Ann. Math. Stud. 102 (1982), 107-131.

[Ji] Z.R. Jin, Liouville theorems for harmonic maps, Invent. Math. 108 (1992), 1-10.

[Ka] M. Kassi, A Liouville theorem for $F$-harmonic maps with finite $F$-energy, Electonic Journal Diff. Equa., Vol. 2006, No. 15 (2006), 1-9.

[Ot1] T.H. Otway, Maps and fields with compressible density, Rend. Sem. Mat. Univ. Padova. 111 (2004), 133-159.

[Ot2] T.H. Otway, Geometric analysis near and across a sonic curve, in: C.V. Bendo (Ed.), New Developments in Mathematical Physics Research, Nova, Science publisher, New York, 2004, pp. 27C45.

[PRS] S. Pigola, M. Rigoli and A.G. Setti, Vanishing and finiteness results in geometric analysis: a generalization of the Bochner technique, Prog. in Math. Vol.266 (2008).

[Se] H.C.J. Sealey, Some conditions ensuring the vanishing of harmonic differential forms with applications to harmonic maps and Yang-Mills theory, Math. Proc. Camb. Philos. Soc. 91 (1982), 441-452.

[Si] J. Simons, Minimal varieties in Riemannian manifolds, Ann. of Math. (2) 88 (1968), 62-105.

[Sim] L. Simon, Asymptotic behaviour of minimal graphs over exterior domains, Ann. Inst. Heri Poincaŕe, (3) 4 (1987), 231-242. 
[SY] R. Schoen, S.T. Yau, Harmonic maps and the topology of stable hypersurfaces and manifolds with non-negative Ricci curvature, Comm. Math. Helv. 51 (1976), $333-341$.

[Xi] Y.L. Xin: Differential forms, conservation law and monotonicity formula, Scientia Sinica (Ser A) Vol.XXIX (1986), 40-50.

[Ya] Y.S. Yang, Classical solutions in the Born-Infeld theory, Proc. R. Soc. Lond. A 456 (2000), 615-640.

School of Mathematical Sciences

And

Laboratory of Mathematics for Nonlinear Science

Fudan University, Shanghai 200433

P.R. China

yxdong@fudan.edu.cn

09110180015@fudan.edu.cn

10110180028@fudan.edu.cn 